\title{
Influencia de la soldadura sobre la rotura por tensiones en tubos de acero inoxidable austenitico del tipo 18-11NB(TP347),
}

\author{
S. GUTIERREZ DE SAIZ-SOLABARRIA ${ }^{1}$, I. GUTIERREZ URRUTIA ${ }^{2}$ y J.M. SAN JUAN² \\ ${ }^{1}$ Dpto. Ciencia de Materiales e Ing. Metalúrgica. Universidad del País Vasco(UPV/EHU). Apdo. 644. Bilbao 48080 y Dpto. Laboratorios. Unidad \\ I+D . Babcock \& Wilcox Spain S.A. (BWE/SEPI). Apdo. 294. Bilbao 48080. \\ ${ }^{2}$ Dpto. Física de la Materia Condensada. Metalurgia Física. Facultad de Ciencias (Campus de Leioa). Universidad del País Vasco (UPV/EHU). \\ Apdo. 644. Bilbao 48080.
}

Se estudia el efecto de la soldadura sobre la rotura por tensiones en tubos de acero inoxidable austenítico estabilizado con $\mathrm{Nb}$ o Ti de los tipos 18-11Nb (TP 347) y 18-11Ti (TP 321) así como sin estabilizar del tipo 18-11 (TP 304) y con Mo del tipo 18-11-2 (TP 316). Los tubos, en todos los casos, son de dimensiones 76,2 mm (3") ф x 2,54 mm (1/10") de espesor y la tensión aplicada (o) de $10 \mathrm{Ton} / \mathrm{in}^{2}$, para una temperatura de servicio de $593^{\circ} \mathrm{C}$.

Palabras clave: Rotura por tensiones, soldadura, aceros inoxidables austeniticos, tubos laminados en caliente.

Influence of welding on stress-cracking failure in type 18-11Nb, 18-11Ti, 18-11 and 18-11-2 austenitic stainless steel tubes

This work has been undertaken to study the stress cracking failure in various 18Cr11Ni [TP347 (18-11Nb, TP 321 (18-11 Ti), TP304 (18-11) and TP316 (18-11-2)] types austenitic stainless steel tubes. The material used was produced hot-rolling at 76,2 $\mathrm{mm} \phi\left(3^{\prime \prime} \phi\right) \times 2,54 \mathrm{~mm}\left(1 / 10^{\prime \prime}\right)$ thickness. The study was carried out at $593^{\circ} \mathrm{C}$ and applied stress of $50 \mathrm{Ton} / \mathrm{in}^{2}$.

Key words : Stress-cracking failure, welding, austenitic stainless steels, hot-rolled tubes.

\section{INTRODUCCIÓN}

Es sabido la problemática que presentan los aceros inoxidables austeníticos (estabilizados con $\mathrm{Nb}$ ) de secciones gruesas, cuando son soldados, dándose en servicio importantes condiciones de restricción. En esta línea el presente trabajo intenta estudiar el efecto de la unión soldada (restricción) sobre la rotura por tensiones de tubos sin soldadura laminados en caliente, a dimensiones de 76,2 $\mathrm{mm}\left(3^{\prime \prime}\right) \phi \times 2,54 \mathrm{~mm}\left(1 / 10^{\prime \prime}\right)$ de espesor. Se aplica a tubos de acero inoxidable austenítico estabilizado con $\mathrm{Nb}$ del tipo $18-11 \mathrm{Nb}$ (TP347), estabilizado con Ti del tipo 18-11Ti (TP 321), sin estabilizar del tipo 18-11 (TP 304) y con Mo del tipo 18-11-2 (TP 316). En todos los casos, presurizamos a $105 \mathrm{Kg} / \mathrm{cm}^{2}\left(1500 \mathrm{lb} / \mathrm{in}^{2}\right)$ para inducir una $\sigma$ total de $10 \mathrm{Ton} / \mathrm{in}^{2}$., asumiendo las expresiones de cálculo que se indican en el código ASME [1].

\section{PROCEDIMIENTO EXPERIMENTAL}

\subsection{Materiales}

Se han empleado tubos de acero sin soldadura, laminados en caliente, de 76,2 mm (3") de $\phi, 2,54 \mathrm{~mm}\left(1 / 10^{\prime \prime}\right)$ de espesor y una longitud de $254 \mathrm{~mm}\left(10^{\prime \prime}\right)$. Los tubos son de acero inoxidable austenítico y cumplen con los grados TP 347, TP 321, TP 304 y TP 316 de la especificación A 312 de la norma ASTM (2) correspondiéndose a su vez con los típicos aceros austeníticos estabilizados $18-11 \mathrm{Nb}(\mathrm{CrNiNb}), 18-11 \mathrm{Ti}(\mathrm{CrNiTi})$ y los sin estabilizar 18-11 (CrNi) y 18-11-2 (CrNiMo), respectivamente. La composición química se ha obtenido utilizando las técnicas
TABLA 1. COMPOSICIÓN QUÍMICA OBTENIDA EN LOS MATERIALES ESTUDIADOS.

\begin{tabular}{|c|c|c|c|c|}
\hline \multirow{3}{*}{ Elemento } & \multicolumn{4}{|c|}{ Composición, \% en masa } \\
\cline { 2 - 5 } & $\begin{array}{c}\mathbf{1 8 - 1 1 N b} \\
(\mathbf{T P ~ 3 4 7 )}\end{array}$ & $\begin{array}{c}\mathbf{1 8 - 1 1 T i} \\
\mathbf{( T P} 321)\end{array}$ & $\begin{array}{c}\mathbf{1 8 - 1 1} \\
\text { (TP 304) }\end{array}$ & $\begin{array}{c}\mathbf{1 8 - 1 1 - 2 ~ M o} \\
\text { (TP 316) }\end{array}$ \\
\hline $\mathbf{C}$ & $\mathbf{0 . 0 8}$ & 0.05 & 0.08 & 0.06 \\
\hline $\mathbf{M n}$ & $\mathbf{1 . 3 0}$ & 1.36 & 1.38 & 1.44 \\
\hline $\mathbf{S i}$ & 0.50 & 0.72 & 0.75 & 0.48 \\
\hline $\mathbf{P}$ & 0.012 & 0.012 & 0.012 & 0.018 \\
\hline $\mathbf{S}$ & 0.007 & 0.006 & 0.009 & 0.007 \\
\hline $\mathbf{C r}$ & 18.72 & 17.63 & 17.74 & 17.65 \\
\hline $\mathbf{N i}$ & 11.90 & 11.87 & 11.25 & 11.17 \\
\hline $\mathbf{M o}$ & 0.03 & 0.15 & - & 2.53 \\
\hline $\mathbf{N b}$ & 1.05 & - & - & - \\
\hline $\mathbf{T i}$ & - & 0.58 & - & - \\
\hline
\end{tabular}

analíticas instrumentales de espectrometría de emisión atómica por descarga eléctrica en alto vacío (AES) y por plasma de acoplamiento inductivo (ICP) y los valores se muestran en la Tabla I.

\subsection{Desarrollo}

Se procede al sellado de extremos mediante tapas hemisféricas que soldamos mediante soldadura por arco protegido por argón (Ar). Después se suelda al exterior del tubo y en la mitad de su longitud un taco o soporte de calidad fundición Calmet $(25 \% \mathrm{Cr}, 12 \% \mathrm{Ni})$ utilizando electrodos Nicrex y soldadura multicapa obteniendo un depósito soldado con un contenido en ferrita del 3-6\%. A continuación presurizamos el conjunto con $\mathrm{N}_{2}$ mediante instalación correspondiente con válvulas de retención y manómetro al efecto y seguidamente 
lo introducimos en un horno de mufla para mantenerlo a la temperatura deseada. Figura 1. Presurizamos a una $p=105$ $\mathrm{Kg} / \mathrm{cm}^{2}\left(1500 \mathrm{lb} / \mathrm{in}^{2}\right)$ y lo mantenemos durante un tiempo continuado a una temperatura de $593^{\circ} \mathrm{C}\left(1100^{\circ} \mathrm{F}\right)$. Asumiendo para el cálculo de $\sigma$ la expresión dada por ASME [1] observamos que las condiciones experimentales citadas imponen al material una $\sigma$ total de $10 \mathrm{Ton} / \mathrm{in}^{2}$.

\section{RESULTADOS Y DISCUSION}

\subsection{Acero $18-11 \mathrm{Nb}$. Soldado y sin soldar al soporte. (Sin/con PWHT).}

Si tenemos en cuenta los resultados obtenidos por otros investigadores [3] y aplicamos la relación " $\sigma$ aplicada $-\mathrm{t}$ a rotura" cabría esperar un tiempo de rotura de aproximadamente 4000 horas (para $\mathrm{T}=593^{\circ} \mathrm{C}$ ) cuando la $\sigma$ que apliquemos sea de $10 \mathrm{Ton} / \mathrm{in}^{2}$. Sin embargo en el presente trabajo observamos que la rotura acaece a un $\mathrm{t}=500$ horas (solo el $12,5 \%$ de lo esperado), para similares condiciones experimentales. Tabla II. El agrietamiento se localiza en la ZAT (Zona Afectada Térmicamente) donde la estructura es austenítica de grano grueso con precipitación de carburos y se inicia en la superficie exterior del tubo progresando a través del espesor con morfología intergranular. Fig. 2 y 3. (Sin PWHT). Con PWHT de solubilización a $1050^{\circ} \mathrm{C}(1 / 2 \mathrm{~h})$ y enfriando al aire el material se agrieta a las $1700 \mathrm{~h}$. Sin soldar el soporte al tubo éste no se agrieta a las 8000 h. Fig. 4.

\subsection{Para los materiales 18-11Ti, 18-11-2 y 18-11. Soldado al} soporte. Sin PWHT.

* Material 18-11Nb. Agrietamiento a las 500 horas $(\approx 21$ días)

* Material 18-11. Agrietamiento a las 3600 horas ( $\approx 150$ días)

* Material 18-11Ti. No se agrieta a las 10500 horas $(\approx 438$ días)

* Material $18-11-2$. No se agrieta a las 14650 horas $(\approx 610$ días)

\subsection{Observación común}

En todos los casos el agrietamiento se inicia en la superficie exterior del tubo donde los esfuerzos de tracción son máximos y se localiza en la ZAT de la unión soldada soporte-tubo donde el grano austenítico es grueso y presenta carburos precipitados en borde de grano. La progresión del agrietamiento, a través del espesor del tubo, es de naturaleza preferentemente intergranular.

\subsection{Precipitados de $\mathrm{NbC}$}

Entendemos que los precipitados de $\mathrm{NbC}$ observados por SEM (microscopía electrónica de barrido, MEB) en la ZAT del acero TP347 $(18-11 \mathrm{Nb})$ y de un tamaño $\leq 200 \AA$ reducen de forma cosiderable las propiedades mecánicas de citado acero, en particular su tenacidad y ductilidad. Resulta eficaz un tratamiento térmico (PWHT) de solubilización de carburos.

\section{CONCLUSIONES}

Como resumen del estudio experimental de comparación de comportamiento de la unión soldada de cuatro aceros inoxidables austeníticos del tipo $18 \mathrm{Cr} 11 \mathrm{Ni}$ frente a una misma
TABLA 2. VALORES OBTENIDOS DE LA RELACIÓN “ $\sigma$-T A ROTURA" EN EL SISTEMA “TUBO SELLADO-SOPORTE SOLDADO SIN PWHT" PARA UNA $\sigma=10$ TON/IN². SIN PWHT.

\begin{tabular}{|c|c|c|c|c|c|}
\hline \multirow{2}{*}{$\begin{array}{l}\text { Material } \\
\text { (Calidad) }\end{array}$} & \multirow{2}{*}{ PWHT } & \multicolumn{2}{|c|}{$T$} & \multirow{2}{*}{$\begin{array}{c}\sigma \text { Aplicada } \\
\left.\text { (ton/ } / \mathbf{i n}^{2}\right)\end{array}$} & \multirow{2}{*}{$\begin{array}{c}\text { t a rotura } \\
\text { (horas) }\end{array}$} \\
\hline & & ${ }^{\circ} \mathbf{F}$ & ${ }^{\circ} \mathrm{C}$ & & \\
\hline $\begin{array}{l}18-11 \mathrm{Nb} \\
(\mathrm{TP} 347)\end{array}$ & NO & 1100 & 593 & 10 & 500 \\
\hline $\begin{array}{l}18-11 \mathrm{Ti} \\
(\mathrm{TP} 321)\end{array}$ & “ & " & “ & “ & $>10.500$ \\
\hline $\begin{array}{c}18-11-2 \\
\text { (TP 316) }\end{array}$ & “ & " & “ & $"$ & $>14.650$ \\
\hline $\begin{array}{c}18-11 \\
\text { (TP 304) }\end{array}$ & NO & 1100 & 593 & 10 & 3.600 \\
\hline
\end{tabular}

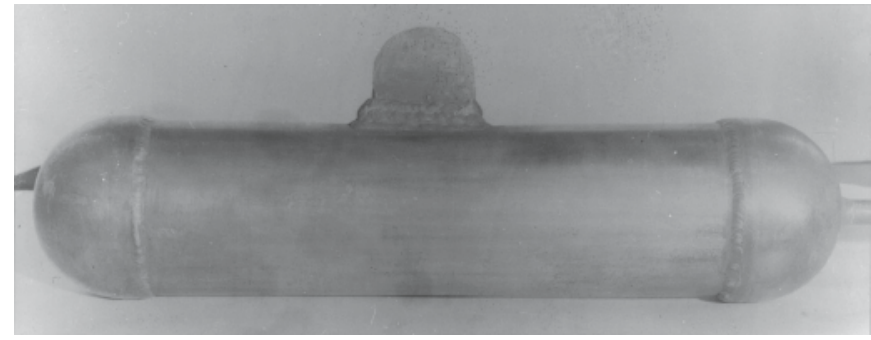

Figura 1. Tubos de 76,2 mm (3") de $\phi, 2,54 \mathrm{~mm}$ de espesor y $254 \mathrm{~mm}$ $\left(10^{\prime \prime}\right)$ de longitud. Sellado de extremos mediante tapas hemisféricas y soporte soldado al exterior. ( $x 0,5)$.

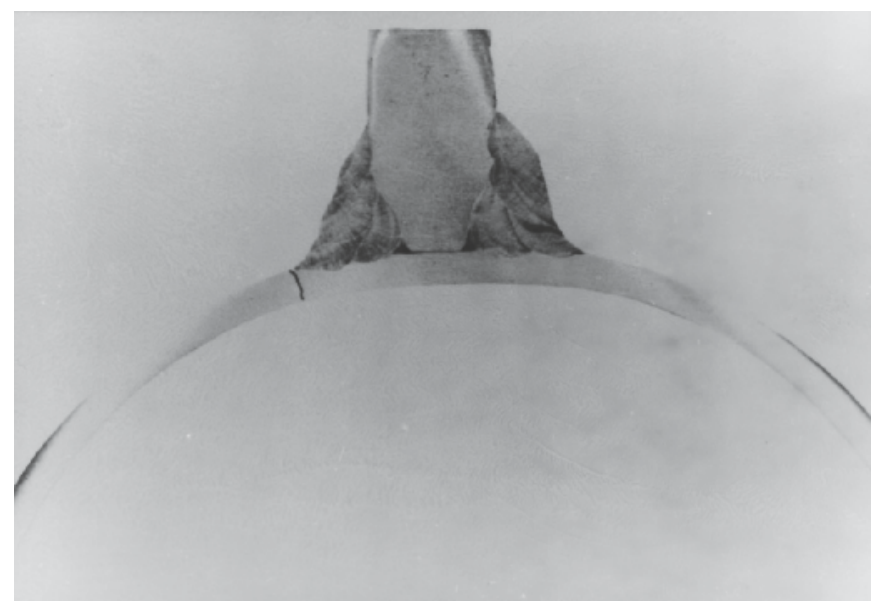

Figura 2. Sección del tubo en calidad 18 - $11 \mathrm{Nb}$ ( TP 347 ) con el soporte soldado. Localización del agrietamiento en la ZAT del tubo.

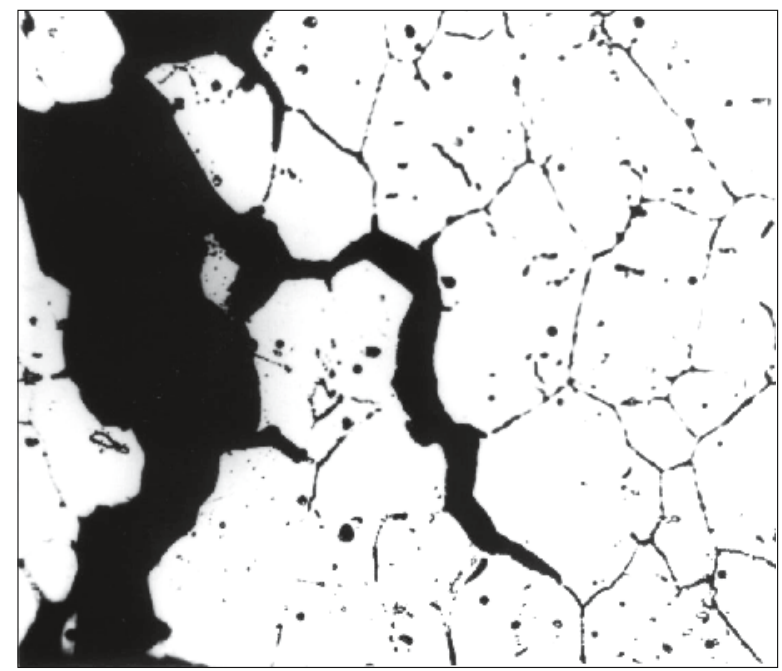

Figura 3. Tubo 18 - $11 \mathrm{Nb}$. ZAT de la unión soldada tubo - soporte. Austenita con precipitación de carburos en límite de grano. Progresión del agrietamiento. ( x 250 ) 


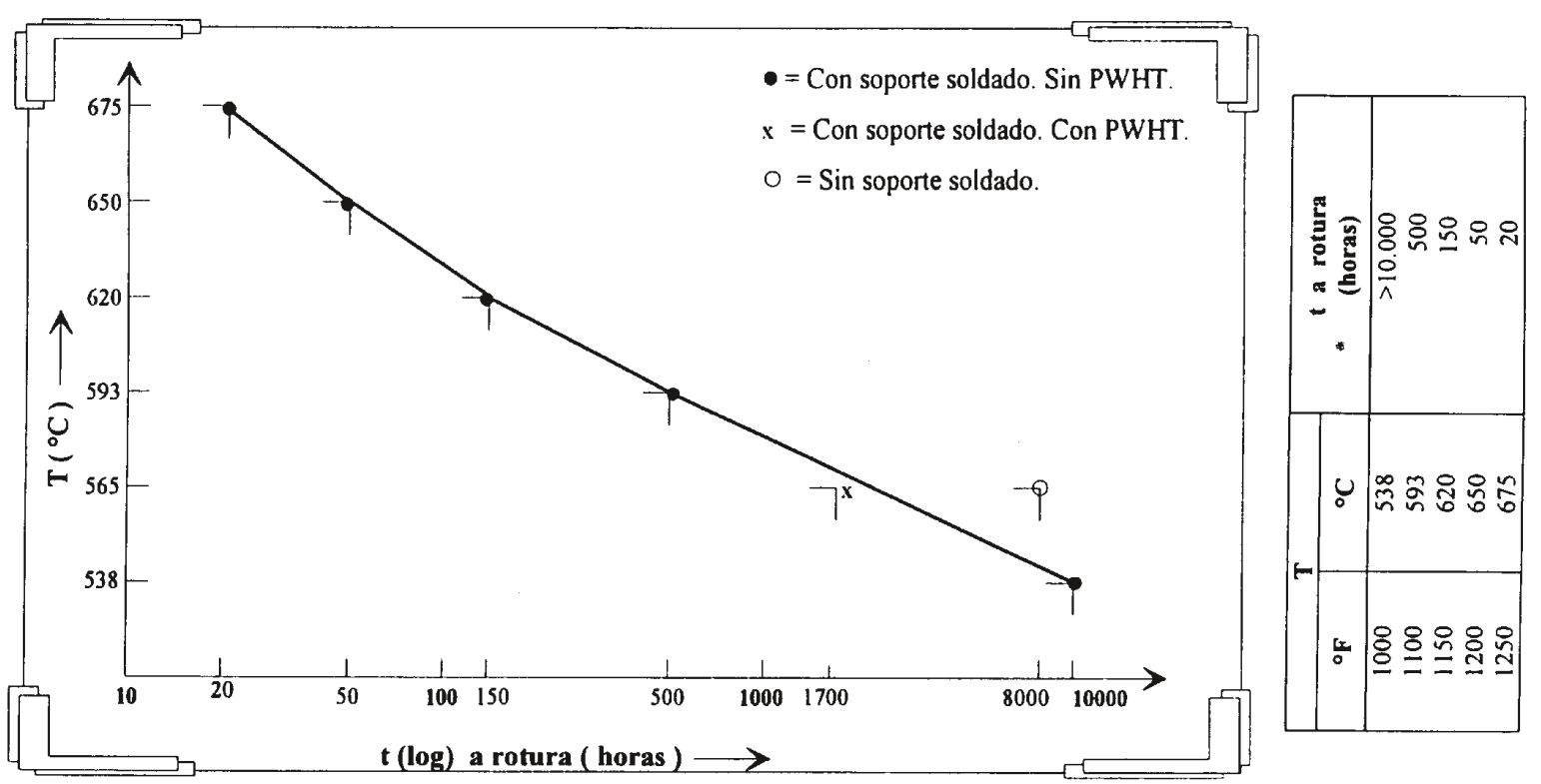

Figura 4. Representación gráfica de “T respecto a t de rotura” y valores obtenidos para el caso a) tubo - soporte y sin PWHT. Para o=10 Ton/in².

tensión de trabajo $\left(\sigma=10 \mathrm{Ton} / \mathrm{in}^{2}\right)$ y temperatura de servicio $\left(\mathrm{T}=593^{\circ} \mathrm{C}\right)$, podemos decir lo siguiente :

i) Se confirma que el peor comportamiento lo presenta la unión soldada del acero austenítico estabilizado con $\mathrm{Nb}$ (18$11 \mathrm{Nb}$ ) del tipo TP347. También observamos que el mejor comportamiento lo presenta la unión soldada del acero inoxidable austenítico con Mo (18-11-2Mo) del tipo TP316.

ii) La ZAT del acero inoxidable austenítico estabilizado con $\mathrm{Nb}$ en calidad $18-11 \mathrm{Nb}$ (TP347) es propensa al fallo por rotura frágil siendo $\sigma$ y $\mathrm{T}$ sus factores determinantes. Los $\mathrm{NbC}$ precipitados en borde de grano reducen de forma considerable la tenacidad y ductilidad del acero.

\section{BIBLIOGRAFIA}

1. ASME B \& PVC. "Rules for construction of pressure vessels". Division I. Section VIII. Código ASME, (1998).

2. ASTM A 312. "Standard specification for seamless and welded austenitic stainless steel pipes". ASTM. Section I. Vol. 01.01. 174-182, (1997).
3. A.J. Perrin and B.P. Parikh. "Raport Confidential". Babcock \& Wilcox Internacional. Mc. Dermott Comp. Barberton. Ohio. USA, (1997).

4. S. Gutierrez . "Efectos de la precipitación causada por la exposición a la temperatura de servicio $\left(600^{\circ} \mathrm{C}\right)$ en un acero austenítico $18-12 \mathrm{Nb}$ después de tratamiento térmico a $900-1300^{\circ} \mathrm{C}^{\prime \prime}$. Soldadura No.24 (4). 190-192. CENIM, (1994).

5. S. Gutierrez y J.M. San Juan. "Influencia de la precipitación causada por la exposición a la T de servicio $\left(600^{\circ} \mathrm{C}\right)$ sobre un acero austenítico 18-11-2 después de un calentamiento por soldadura a $900-1350^{\circ} \mathrm{C}^{\prime \prime}$. Soldadura No. 25 (2). 82-86. CENIM, (1995).

6. S. Gutierrez, J.M. San Juan, A. Valea y A.G. Hidalgo. "Efecto de la precipitación causada por exposición a la temperatura de servicio $\left(600^{\circ} \mathrm{C}\right)$ de un acero austenítico "Esshete 1250" después de varios tratamientos térmicos en el rango $700-1250^{\circ} \mathrm{C}^{\prime \prime}$. VI Congreso Nacional de Tratamientos Térmicos y de Superficie, (1995).

7. S. Gutierrez , I. Gutierrez Urrutia y J.M. San Juan . "Situación actual de la tecnología de los aceros inoxidables austenoferríticos duplex y superduplex". Q.e I..No. 2. 22-32, (1999).

8. J.R. Davis \& ASS. "Stainless Steels". ASM. Especialty Handbook. OH. USA, (1996).

9. S.S. Hecker et al. “Effects of strain State ad Strain Rate on Deformation-Induced Transformation in 304 Stainless Steel". Part. I and II. Metall. Trans A. Vol 13 A, (1992).

10. K.G. Caroll et al. “Chromium Destribution around Grain Boundery Carbides found in Austenitic Stainless Steel". Nature. Vol. 184. 1959.

11. ASM Handbook. "Heat Treating of Stainless Steels". Heat Treating. Vol 4, (1991).

12. D.J. Kotecki. "Welding of Stainless Steels". Welding, Brazing and Soldering. ASM Handbook. ASM Int'. Vol 6, (1993). 